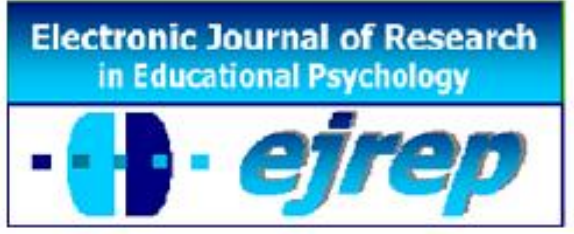

\title{
Tipología de Actividades Extraescolares y Procrastinación Académica en Alumnado de Educación Primaria
}

\section{Clariana, M. ${ }^{1}$, Cladellas, R. ${ }^{1}$, Gotzens, C. $^{2}$, Badia, M. ${ }^{1}$ y Dezcallar, T.}

${ }^{1}$ Facultat de Psicología, Universitat Autònoma de Barcelona UAB, Barcelona ${ }^{2}$ Facultat de Psicología, Universitat de les Illes Balears UIB, Palma de Mallorca

\section{España}

Correspondencia: Mercè Clariana. Facultat de Psicologia, Universitat Autònoma de Barcelona, Bellaterra 08193, Barcelona, España. E-mail: merce.clariana@uab.cat

(C) Education \& Psychology I+D+i and Ilustre Colegio Oficial de la Psicología de Andalucía Oriental 


\section{Resumen}

Introducción. Algunos trabajos anteriores han demostrado que la participación en actividades extraescolares optimiza tanto las notas académicas de los alumnos como sus procesos de socialización. Sin embargo, ninguno ha relacionado las actividades extraescolares con la tendencia a la procrastinación académica, o sea la costumbre, más o menos arraigada, de dejar las tareas de estudio para más adelante. El trabajo que ahora presentamos relaciona estas dos variables y resulta una primicia en este campo.

Método. Se han recogido cuestionarios contestados por padres de un total de 417 alumnos y alumnas de los ciclos medio y superior de educación primaria (de 8 a 13 años). Para identificar la participación en extraescolares se han utilizado dos parámetros: la tipología de las actividades y los años de experiencia de los niños y niñas en la realización de las mismas. Para la procrastinación académica se ha utilizado una escala de 0 -no procrastina- a 10 -procrastina mucho o siempre-.

Resultados. Los resultados indican que tanto la tipología de actividades extraescolares, como los años de experiencia en las mismas, y también el nivel de procrastinación académica percibido por los padres, muestran pautas claramente diferenciadas entre niños y niñas. Además, los alumnos de género masculino mejoran la procrastinación académica reportada por los padres partir de 4 años de experiencia en extraescolares de deporte; en cambio, las niñas controlan mejor la procrastinación percibida por los padres a partir de 4 años de experiencia en extraescolares de tipo artístico.

Discusión y conclusión. Los resultados son, en general, coherentes con trabajos anteriores y, además, amplían el conocimiento al tratar conjuntamente la procrastinación y las actividades extraescolares y, también, al introducir la diferenciación del género, que permite diseñar escenarios de actividades extraescolares más personalizados para cada uno de los alumnos y alumnas durante la educación primaria.

Palabras Clave: actividades extraescolares o extracurriculares, procrastinación académica, educación primaria, ciclo medio de primaria, ciclo superior de primaria, género. 


\title{
Typology of extracurricular activities and academic procrastination in primary education students
}

\begin{abstract}
Introducción. Previous works have shown that participation in extracurricular activities optimize both academic grades and socialization processes of the students. However, none of them has related extracurricular activities with the tendency to procrastinate in academic environments, that is, the more or less usual habit of leaving important school duties for later without a reason. The study presented here connects these two variables and is a first in this field.
\end{abstract}

Método. Questionnaires completed by parents of 417 students from the middle and upper primary education were collected. To identify participating in extracurricular activities two parameters have been used: the type of activities and the years of children's experience in practising them. For academic procrastination we designed a scale asking parents to rate this habit of their children in any point between 0 -he or she doesn't procrastinate- to 10 -he or she always procrastinates or always procrastinates when doing school activities-.

Resultados. Results indicate that either the types of extracurricular activities or the level of the students procrastination, show distinct patterns between boys and girls. Male students improve their school procrastination management from 4 years of experience in extra school sport activities; in contrast, girls have better academic procrastination control from 4 years of experience in extracurricular artistic type activities.

Discusión y conclusion. The outcomes of the present article are generally consistent with previous results in the procrastination and extraschool activities field and also extend the knowledge by introducing gender differentiation, which allows designing extracurricular activities scenarios more suited and customized for every one of the pupils in primary education.

Palabras Clave: extra school or extracurricular activities, academic procrastination, primary school, children aged 8-10, children aged 10-12, gender. 


\section{Introducción}

Este estudio se ha inspirado en un buen número de trabajos anteriores, realizados mayoritariamente a partir de la década 2000-2010, que han informado sobre las consecuencias y beneficios que las actividades extraescolares aportan a los alumnos. Con todo, ninguna de las investigaciones publicadas hasta la fecha ha relacionado las actividades extraescolares con la tendencia a la procrastinación académica, que es la demora innecesaria en la realización de las tareas de los estudios, y que incide tanto en la confianza y seguridad de los estudiantes como en su aprovechamiento en el ámbito educativo.

Por ello, la presente investigación pretende analizar la relación entre estas dos variables, con la intención de identificar conexiones entre ellas, que serán de ayuda para padres, docentes y psicólogos educativos a la hora de tomar decisiones sobre el tipo de extraescolares que se pueden aconsejar a los alumnos y alumnas de primaria, y a los beneficios relacionados con el control de la procrastinación que se pueden esperar de la participación en estas actividades.

\section{Actividades extraescolares}

La participación en actividades extraescolares o extracurriculares se ha popularizado en las sociedades occidentales (Moriana et al., 2006), ya sea porque los padres valoran positivamente que sus hijos estén sujetos a un horario y a un proceso instruccional también fuera de la escuela (Osgood, Anderson y Shaffer, 2005), o por las mayores oportunidades de formación y relación social que estas actividades ofrecen (Mahoney y Vest, 2012). Recientemente, algunos autores han demostrado que dicha participación, entre los 5 y los 18 años de edad, abarca el $80 \%$ de la población y alcanza una media de unas 5 horas a la semana (Cladellas, Clariana, Badia y Gotzens, 2013; Fredricks 2012; Mahoney, Harris y Eccles, 2006).

Además, son diversos los expertos que han probado los efectos beneficiosos de las actividades extraescolares, en áreas también diversas de la vida de los alumnos. Al respecto de este punto, y en cuanto a las extraescolares en general, se ha informado, por ejemplo, de que inciden en un mejor desarrollo de las actitudes ciudadanas y cívicas (Keser, Akar y Yildrim, 2011), incrementan la autorrealización y la autoestima (Ritchie y Williamon, 2011), previenen 
los conflictos durante la adolescencia (Mahoney y Vest, 2012), contribuyen a la consecución de notas académicas más altas (Cladellas et al., 2013; Mahoney et al., 2006; Moriana et al., 2006), y procuran efectos positivos de larga duración, que se pueden observar incluso después de 2 a 4 años de finalizada su realización (Fredricks, 2012).

A pesar de estos buenos resultados, otros investigadores han hecho hincapié en lo que se ha denominado la Over-Scheduling Hypothesis, refiriéndose al peligro de sobrecargar a los niños con excesivas actividades fuera de la escuela, lo que en lugar de procurar ventajas sólo acarrearía inconvenientes (Elkind, 2001; Luthar, Shoum y Brown, 2006; Marsh y Kleitman, 2002). Para desmentir esta idea se ha comprobado que, de hecho, las extraescolares se relacionan con los beneficios que provocan no de una manera lineal sino de acuerdo a una curva con forma de $\mathrm{U}$ invertida, y que superadas 5 actividades extraescolares simultáneas distintas y 10 horas totales a la semana, el efecto para el alumno ya es significativamente perjudicial en lugar de resultar beneficioso (Bennett, Lutz y Jayaram, 2012; Cladellas et al., 2013; Fredricks, 2012; Mahoney y Vest, 2012).

Así, en el momento actual los autores más relevantes en este campo están de acuerdo en que una dedicación contenida a las actividades extraescolares refuta el efecto negativo de la Over-Scheduling y que, en términos generales, es adecuado involucrar a los niños y adolescentes en actividades extraescolares si se dedican moderadamente a las mismas. Con todo, más allá de la Over-Scheduling no tenemos noticia de ningún trabajo que haya relacionado la participación en extraescolares específicamente con el control, planificación y regulación del tiempo por parte de los alumnos. Este aspecto llama la atención principalmente porque, a nuestro modo de ver, una vez aceptado que las extraescolares ocupan el tiempo libre de los alumnos, sería de sumo interés evaluar como organizan el tiempo restante y su dedicación a los estudios los niños y niñas que en dichas actividades participan.

Paralelamente, en cuanto a la identificación y medida de la participación en extraescolares, se ha indicado que sería necesario fijar la atención en alguno de estos aspectos (Bonhert, Fredricks, y Randall, 2010): 1. Breath, amplitud, variedad o tipo de actividades extraescolares que el alumno realiza a la semana, 2. Intensity, intensidad o cantidad de horas a la semana de extraescolares de cualquier tipo, 3. Duration, duración o años de "antigüedad" en la participación en cada tipo de extraescolares, y finalmente 4. Engagement, compromiso o motivación 
intrínseca del alumno -y no de los padres (Trost, Biesecker, Stattin, y Kerr, 2007)- en dichas actividades.

Por otro lado, algunos expertos informan de los efectos de las actividades extraescolares según la tipología de las mismas. Acerca de este punto, las actividades fuera del horario escolar que han suscitado más estudios, quizás por ser las más concurridas, son las de deportes y actividad física, que se han relacionado con un mayor bienestar general de los alumnos que las practican (Dimech y Seiler, 2011; McHale et al., 2005), prevención de la delincuencia (Gardner, Roth y Brooks-Gunn, 2011), mejores notas escolares (Cladellas et al., 2013; O’Connor y Jose, 2012) y mayores oportunidades para la relación social (Schaefer, Simpkins, Svest y Price, 2011), por citar solo algunos trabajos recientes. Igualmente, se han publicado artículos que llaman la atención sobre la pertinencia de las actividades extraescolares artísticas y musicales para potenciar aspectos relacionados por ejemplo con un mejor desarrollo de la noción de tiempo y espacio (Atkinson y Robson, 2012), de la memoria verbal (Ho, Cheung y Chan, 2003), o de la autoestima y la satisfacción personal (Ritchie y Williamon, 2011), también por citar únicamente una muestra de los estudios realizados en los últimos años.

Para acabar, cabe destacar que hay cierta controversia cuando se trata de distinguir entre alumnos de género masculino y femenino para especificar mejor las ganancias que las actividades extraescolares en general y distintos tipos de las mismas aportan. Hay expertos que indican no haber encontrado ninguna relación significativa entre el género del alumno y el tipo de extraescolares en que participa (Arisoy y Tutkun, 2012) mientras que otros, han puesto de relieve una idiosincrasia claramente diferenciada entre géneros en este aspecto (McCoy, Byrne y Banks, 2012). La polémica en cuanto al género, pues, está servida, por lo que parece de interés incorporar este aspecto en presentes y futuras investigaciones relacionadas con las actividades extraescolares y los beneficios que conllevan.

\section{Procrastinación}

De acuerdo a Steel y Ferrari (2013), probablemente los autores actualmente más destacados en este campo, la procrastinación es un fallo en los procesos de autorregulación o - en lenguaje más llano- es el hábito de dejar para mañana lo que se debe y se puede hacer hoy, sin que exista una causa objetiva que explique la demora (Lay, 1997). Esta característica se ha venido estudiando en psicología desde la década de los ochenta y ha demostrado ser claramente perjudicial para el aprendizaje académico, tal y como comentan algunos autores desde 
distintas latitudes (Clariana, Gotzens y Badia, 2011; Clariana, Gotzens, Badia y Cladellas, 2012; Lakshminarayan, Potdar y Reddy, 2013; Lubbers, Van Der Werf, Kuyper y Hendriks, 2010; Moon y Illingworth, 2005, Rothblum, Solomon y Murakami, 1986).

Además de acompañar a las notas académicas bajas, la procrastinación presenta otras implicaciones negativas, por ejemplo, estar relacionada con un nivel también más bajo de consciencia como factor de personalidad (Steel, 2007), y con un nivel de neuroticismo (Özer, 2012) e impulsividad más altos (Schouwenbourg y Lay, 1995; Steel y Ferrari, 2013); correlacionar negativamente con la utilización de estrategias metacognitivas y de autorregulación durante el aprendizaje (Howell y Watson, 2007); estar conectada con un mayor nivel de malestar personal (Rice, Richardson y Clark, 2012); y estar asociada con una menor motivación intrínseca en relación a los estudios (Schouwenbourg y Groenewoud, 2001). Esto por mencionar solo algunas de las particularidades más habitualmente relacionadas con esta tendencia.

Simultáneamente, algunos autores (de Bruin et al., 2012; Dewitte y Lens, 2000; Rhodes y Dickau, 2012) describen la procrastinación como un problema de falta de voluntad combinado con un déficit en el control del tiempo a la hora de realizar las tareas. Es lo que se ha denominado intention-gap dissonance, un constructo que asume que las personas que procrastinan hacen planes y previsiones como las demás, pero no cumplen con sus objetivos porque, entre otras razones, a la hora de la verdad les falta tiempo para hacerlo. En la misma línea, al referirse a los inconvenientes de la procrastinación en el ámbito académico, otros expertos (Ferrari, Johnson y McCown, 1995) ya se habían centrado con anterioridad en el control del tiempo, al afirmar que debido a su costumbre de empezar las tareas escolares siempre tarde, los procrastinadores no tienen tiempo suficiente para realizarlas correctamente, con lo que suelen rendir por debajo de sus posibilidades.

También se ha indicado que los alumnos universitarios que presentan una mayor intention-gap dissonance, actúan apremiados por su propia experiencia en el pasado, que les ha enseñado, o acostumbrado, a empezar las tareas más tarde de lo que sus propósitos iniciales habían previsto. En este sentido, se ha demostrado (por ejemplo Sommer, 2011) que los hábitos y conductas del pasado afectan significativamente a las intenciones y conductas del momento actual. Tal como afirma Sommer en un trabajo reciente (2013) sobre procrastinación y preparación de exámenes: "According to this, repeated behavior (routine) -in other words: experience with regard to behavior- leads to an increase of significance of implementation 
intention with regard to behavior implementation (p. 623). Con todo, en el mismo trabajo el autor, también admite que la distancia entre la intención y la conducta se ve asimismo condicionada, de modo substancial, por la experiencia que el alumno tiene en el momento actual, con lo que, lógicamente, los hábitos del pasado no son la única causa que explica las características del comportamiento en el presente.

Por otra parte, la procrastinación académica o demora infundada en la realización de las tareas de estudio es un hábito que afecta alrededor del $80 \%$ de los alumnos de educación secundaria y universidad (Steel y Ferrari, 2013), etapas en las que las obligaciones con fecha límite y establecidas en un calendario prefijado son bien habituales. Quizás es por esta razón, que más de un tercio de la población general considera que la procrastinación ha significado un inconveniente importante para el correcto afrontamiento de sus retos académicos y educativos (Steel, 2011).

Por lo que se refiere a la escuela primaria en cambio, la procrastinación no ha sido tan estudiada, pues la mayoría de autores defienden que sus efectos nocivos se empiezan a notar a partir de la educación secundaria (Clariana, 2008; Steel, 2007). En primer lugar, actualmente no existen escalas o cuestionarios estandardizados para evaluar la procrastinación académica a esta edad. Además, se ha puesto de relieve que el control de la responsabilidad en la puntualidad de las tareas lo ejercen primero los padres en educación infantil, y después los maestros en educación primaria, donde transmiten dicho control al alumno para que pueda llegar a ser dueño y señor de sus acciones durante la secundaria y la universidad (Tuckman, 1991). También hay quien afirma que, en los niveles más altos de la educación, como la secundaria y la universidad, la tendencia a la procrastinación es menor (Ferrari, Özer y Demir, 2009). Sin embargo, este extremo se ha desmentido, en parte, al demostrar que, en nuestro país, los alumnos de primer año de universidad procrastinan significativamente más que los de ESO, y menos que los que están a punto de acabar la carrera (Clariana et al., 2012).

Asimismo, hay que añadir que además de estos estudios, que han arrojado afirmaciones relacionadas con el desarrollo de los alumnos y la procrastinación a nivel general, únicamente hemos encontrado un trabajo que se refiere a la demora infundada en alumnos de 6 a 11 años. Ya en 1998, Lay, Kovacs y Danto corroboraban que a esta edad la procrastinación es inversamente proporcional a la consciencia, tal como sucede en etapas posteriores, y que también resulta un inconveniente para el aprendizaje en la educación formal. Y, con alumnos de 
mayor edad, de 11 a 17 años, se ha demostrado que la procrastinación se da con más frecuencia en hijos de familias con un nivel sociocultural bajo (Rosário et al., 2009), y también que se observa más habitualmente en niños criados en hogares caracterizados por la aplicación de una disciplina exageradamente estricta (Xin-yi Ling, Zhang, Xiong y Xin-li, 2011).

Para finalizar, es necesario hacer notar que los autores que estudiaron a alumnos de educación primaria (Lay, Kovacs y Danto, 1998) destacaron que ni la consciencia ni la procrastinación en edad escolar están relacionadas con el género del alumno, una información que es contradictoria con la mayoría de estudios posteriores sobre procrastinación en alumnos de más edad, que indican reiteradamente que los estudiantes varones demoran sin motivo más habitualmente que las chicas (Steel, 2007, 2010; Steel y Ferrari, 2013). En cuanto a esta diferencia en procrastinación relacionada con el género del alumno, no tenemos noticia de otros trabajos llevados a cabo en educación primaria, por lo que parece interesante abordar esta cuestión.

\section{Objetivos}

Puesto que la procrastinación es una tendencia o un hábito que implica el control del tiempo, parece plausible hipotetizar que puede estar relacionada con la participación de los alumnos en actividades extraescolares, pues dichas actividades alteran la cantidad de tiempo libre durante la etapa escolar. Además, se ha demostrado que la conducta en el pasado condiciona la conducta en el momento presente, por lo que es adecuado estudiar los hábitos de procrastinación en alumnos cuando son bien jóvenes, ya que su tendencia a la demora injustificada en una edad temprana podrá afectar a su conducta académica también en el futuro.

Por otra parte, como ya hemos dicho, a menudo se han relacionado las extraescolares deportivas y artísticas con el aprovechamiento académico y el bienestar psicológico y social de los alumnos, pero poco o nada se ha dicho sobre su relación con la procrastinación académica, que directa o indirectamente también incide en estos factores.

Por todo ello, el trabajo que ahora presentamos se plantea los siguientes objetivos:

1. Describir el nivel de procrastinación, la tipología de extraescolares -deporte o artey los años de participación en dichas actividades (o "duración", siguiendo a Bonhert et al, 2010) en niños y niñas de ciclo medio y superior de primaria. 
2. Relacionar estas tres variables, procrastinación académica, tipo, y duración de actividades extraescolares.

3. Estudiar las posibles desigualdades observadas entre géneros también en estas tres variables y detectar influencias mutuas y diferenciales entre las mismas.

\section{Método}

\section{Participantes}

Para llevar a cabo este trabajo, durante el segundo semestre del curso escolar 2011-2012 se pidió la colaboración de 18 centros seleccionados aleatoriamente de escuelas de primaria, públicas (50\%) y concertadas (50\%), de la provincia de Barcelona (43\%) y de la isla de Mallorca (57\%), ambas en España. Los padres de un total de 721 alumnos participaron en la investigación, de los cuales se desestimaron los datos de los alumnos de ciclo inicial por considerar que los niños eran demasiado jóvenes como para tener una experiencia mínimamente sólida y continuada en actividades extraescolares.

De este modo, la muestra del trabajo quedó en padres de 417 alumnos, 206 niños (49\%) y 211 niñas $(51 \%)$ de los ciclos medio y superior de educación primaria, con edades comprendidas entre los 8 y los 13 años (media = 10.01 años; desviación típica $=1.24)$. La prueba de comparación de medias de la edad según el género del alumno no da un resultado significativo, $t=1.27, p=.20$, ni tampoco la de distribución de niños y niñas por curso, $X^{2}=$ $4.19, p=.24$, por lo que se asume que el género, que será una variable importante en el presente trabajo, está distribuido equilibradamente entre los participantes.

\section{Instrumentos y medidas}

Se elaboró un cuestionario ex profeso destinado a los padres o tutores legales de los alumnos de la muestra, cuyo contenido fue validado por un grupo de 15 expertos independientes, formado por docentes de escuela primaria y por profesores universitarios. Ya que la edad mínima de los participantes era de 8 años se consideró que era mejor que los padres contestaran las preguntas pues, como es sabido, en el ciclo medio de primaria la lectoescritura está todavía en proceso de consolidación. Esta práctica, consistente en utilizar a los padres y tutores legales para recabar datos durante la infancia, ya ha sido aplicada con éxito en trabajos 
anteriores (Bode y Hirner, 2013; Gawrolow, Morgenroth, Schultz, Oettingen y Gollwitzer, 2013; Lachar y Gruber, 2001), y por este motivo y también porque no existen escalas de medida de la procrastinación académica en estas edades decidimos adoptarla también en nuestro estudio.

El cuestionario incluía preguntas acerca de los datos socioeconómicos, el curso actual del alumno, sus notas académicas y su experiencia en extraescolares, y también preguntaba sobre procrastinación. En cuanto a este punto, la pregunta valoraba el nivel o grado de procrastinación que los padres percibían en sus hijos, y decía: La procrastinación o remolonería escolar (aquí utilizamos "romanceria", que es una palabra catalana de uso corriente y que significa exactamente procrastinación) es la costumbre de dejar para mañana o para más adelante las tareas de estudio -estudiar, hacer deberes, leer...- sin que exista un motivo real que justifique esta demora. En una escala de 0 a 10 puntúa el nivel de remolonería escolar de tu hijo o hija. En esta escala, 0 significaba no procrastina y 10 procrastina siempre o mucho, de modo que a más puntuación, mayor nivel de procrastinación académica percibida por los padres de los alumnos.

En cuanto a las actividades extraescolares que estaban realizando los alumnos en el momento de la recogida de datos y las que dichos alumnos habían realizado con anterioridad, se utilizó el registro reproducido en la Figura 1 (Anexo).

Siguiendo a los autores antes mencionados (Bonhert et al., 2010), se han utilizado dos medidas para evaluar la participación en extraescolares. Por un lado se ha identificado el tipo de extraescolares que habían realizado los alumnos y por el otro, se ha considerado la duración, antigüedad o años de experiencia en las mismas. Como puede observarse en la hoja de registro, para computar este último aspecto se desestimaron las extraescolares del curso 2011-2012, pues la experiencia de los niños era demasiado breve para ser tenida en cuenta, y se sumaron los cursos enteros anteriores en cada tipo de actividad. Es decir, se acumularon los años de experiencia en actividades extraescolares deportivas, artísticas, otras y totales. Por este motivo, no es de extrañar que en la muestra se encuentren, por ejemplo, niñas que con 9 años de edad ya sumen 13 años de extraescolares totales, si han hecho 5 años de música, 5 de ballet y 3 de inglés. Queremos decir que la duración o suma total de años o cursos de experiencia en extraescolares puede sobrepasar ampliamente la edad de los alumnos, si han participado de forma continuada en actividades simultáneas. 
Por consiguiente, las variables que se han analizado en el presente estudio son: género del alumno, edad, tipo de escuela, duración o experiencia en actividades extraescolares, y procrastinación académica percibida por los padres. Dichas variables se han utilizado del siguiente modo:

- Género. Variable independiente dicotómica de dos categorías, niño y niña.

- Edad. Variable independiente dicotómica medida en relación al ciclo de primaria que cursan los alumnos, en este caso con dos categorías, ciclo medio y ciclo superior.

- Tipo de escuela. Variable independiente dicotómica de dos categorías: pública y concertada.

- Tipología y duración de la participación en actividades extraescolares.

- Arte. Años de experiencia en extraescolares de tipo artístico (siguiendo a Atkinson y Robson, 2012). Ésta es una variable independiente continua con un rango en nuestra muestra de 0 a 13 años. Más adelante esta variable se ha convertido a ordinal, tal como se explicará en el apartado de resultados.

- Deporte. Años de experiencia en extraescolares de tipo deportivo (de acuerdo a O’Connor y Jose, 2012, entre otros autores). Ésta es una variable independiente continua que presenta un rango de 0 a 14 años de antigüedad. Igualmente, se ha convertido en ordinal más adelante.

○ Otras. Años de experiencia en todas las actividades extraescolares que no están incluidas en las dos categorías anteriores. Esta variable es asimismo independiente continua, con un rango de 0 a 11 años de práctica, e incluye extraescolares de idioma, cocina, cultura popular, ajedrez, escultismo u otras asociaciones infanto-juveniles, informática, magia y jazz. En el curso del análisis, esta variable se ha convertido igualmente de continua a ordinal.

- Actividades extraescolares totales. Esta variable independiente continua es la suma o acumulación de los años de todas las actividades extraescolares en que han participado los alumnos de ciclo medio y superior de educación primaria, o sea es la suma de las variables experiencia en extraescolares de arte, deporte y otras. Las extraescolares totales permiten apreciar los beneficios o inconvenientes (de acuerdo a la Over-Scheduling Hypothesis) que un alumno obtiene de las actividades fuera de la escuela en general (Mahoney y Vest, 2012). En la muestra que presentamos las 
extraescolares totales tienen un rango de 0 a 16 años, un valor que más adelante también se ha convertido a ordinal como en las demás variables independientes.

- Procrastinación. Percepción de los padres de la demora injustificada en la realización de las tareas escolares, valorada en una escala de 0 a 10 puntos. Esta es la variable dependiente y continua del presente estudio de modo que, hipotéticamente, la tendencia a la procrastinación percibida por los padres puede ser distinta entre alumnos y alumnas, y también puede variar en función del tipo y la duración de las actividades extraescolares en que los niños y niñas participan o han participado.

\section{Procedimiento}

En una primera fase, el equipo de investigadores se reunió con la dirección de los centros seleccionados con la intención de explicar el objetivo del trabajo. En algunos casos, y a solicitud del propio equipo directivo del centro, fue necesario proporcionar una carta de explicación de la investigación, para que ésta fuera aprobada por la asociación de madres y padres del correspondiente centro. En la reunión con los docentes también se les informó de que se administraría un cuestionario con varias preguntas a los padres de los niños.

A continuación, la propia dirección de cada uno de los centros participantes era la encargada de proporcionar los cuestionarios a los alumnos para que éstos, a su vez, los entregaran a sus padres, que eran quienes debían responder a las preguntas planteadas. Los padres disponían de un tiempo máximo de dos semanas para devolver los cuestionarios debidamente cumplimentados. Estos tenían instrucciones de entregarlos a la secretaría del centro escolar de su hijo/a. Los cuestionarios que no se recibieron en el plazo establecido fueron excluidos de la muestra.

\section{Análisis estadístico}

Para los análisis estadísticos se ha utilizado el paquete IBM SPSS Statistics Version 19. En primer lugar, con la prueba $t$ de Student, se han calculado las diferencias entre niños y niñas en las siguientes variables cuantitativas: años de experiencia en extraescolares de arte, deporte, otras y totales, y nivel de procrastinación académica de los alumnos percibido por los padres. A la vista de los resultados significativos que se han encontrado para la mayoría de los aspectos estudiados se decidió proceder aplicando los análisis posteriores separadamente según el género de los alumnos. 
En segundo lugar, se han convertido las variables independientes de continuas a ordinales de dos grupos, uno denominado bajo para las puntuaciones situadas por debajo del percentil 50 y el otro denominado alto para los valores que se encuentran por encima de dicho percentil. Seguidamente, se han aplicado pruebas $t$ de Student a los niveles de procrastinación académica percibida por los padres según las nuevas variables ordinales creadas, referidas al tipo y duración de las extraescolares. Como decimos, todos estos cálculos se han realizado separadamente para niños y niñas. Cabía la posibilidad de aplicar MANOVAs controlando para el género, pero los resultados de la interacción no eran claros y hemos preferido hacer los análisis separados para apreciar más directamente y nítidamente la influencia de las variables independientes en la procrastinación que perciben los padres de los alumnos y alumnas de ciclo medio y superior de primaria.

\section{Resultados}

La descripción y comparación por géneros de las variables continuas del presente trabajo se encuentran en la Tabla 1, que revela que los niños y niñas de ciclo medio y superior de educación primaria presentan valores significativamente distintos en todas las variables estudiadas, excepto en la experiencia en otras actividades extracurriculares aparte de deportes y arte. Por este motivo, y principalmente porque la variable dependiente procrastinación percibida por los padres también muestra diferencias significativas según el género, decidimos continuar los análisis estadísticos separadamente para los niños y las niñas. Asimismo, la Tabla 1 pone en evidencia que los niños realizan significativamente más extraescolares de deporte que las niñas, más extraescolares en total, y que también son reportados como más procrastinadores que ellas. Por su parte, las niñas son percibidas como más puntuales, o sea los padres o tutores dicen que procrastinan menos que sus pares del género masculino, y realizan significativamente más actividades extraescolares de arte, y menos deportivas y totales que ellos. 
Tabla 1. Descripción de las variables continuas -años de extraescolares y procrastinación- y comparación por género con la $t$ de Student $(n=417)$.

\begin{tabular}{lccccc}
\hline $\begin{array}{l}\text { Variables } \\
\text { continuas }\end{array}$ & $\begin{array}{c}\text { Niños } \\
n=206\end{array}$ & $\begin{array}{c}\text { Niñas } \\
n=211\end{array}$ & $t$ & $d$ & $p$ \\
\hline Arte & .79 & 1.62 & 3.63 & .83 & $.00^{*}$ \\
\hline Deporte & 3.71 & 1.49 & 8.57 & 2.22 & $.00^{*}$ \\
\hline Otras & 1.73 & 2.02 & 1.34 & .30 & .27 \\
\hline Totales & 6.23 & 5.14 & 2.26 & 1.09 & $.03 *$ \\
\hline Procrastinación & 6.25 & 5.59 & 4.09 & .67 & $.00^{*}$ \\
\hline$* p<.05$ & & & & &
\end{tabular}

Seguidamente, como ya se ha indicado, se convirtieron las variables independientes años de experiencia en extraescolares de arte, deporte, otras y totales, de continuas a ordinales, tomando como referencia el percentil 50 de las mismas, separadamente por géneros. Al final, estas variables quedaron tal como se expone en la Tabla 2.

Tabla 2. Grupos según los años de experiencia en extraescolares de distinto tipo, de acuerdo al percentil $(n=417)$.

\begin{tabular}{lcccccc}
\hline & Grupo & Percentil & $\begin{array}{c}\text { Años de } \\
\text { arte }\end{array}$ & $\begin{array}{c}\text { Años de } \\
\text { deporte }\end{array}$ & $\begin{array}{c}\text { Años de } \\
\text { otras }\end{array}$ & $\begin{array}{c}\text { Años de } \\
\text { totales }\end{array}$ \\
\hline $\begin{array}{l}\text { Niños } \\
\mathrm{n}=206\end{array}$ & Bajo & $<50$ & 0 & $0-3$ & $0-3$ & $0-6$ \\
\hline Alto & $>50$ & + de 0 & + de 3 & + de 3 & + de 6 \\
$\begin{array}{l}\text { Niñas } \\
\mathrm{n}=211\end{array}$ & Bajo & $<50$ & $0-3$ & $0-3$ & $0-3$ & $0-7$ \\
\hline
\end{tabular}

Como hemos comentado, dadas las diferencias significativas entre géneros presentadas en la Tablas 1 y 2, se procede diferenciadamente entre géneros a partir de ahora, para analizar la comparación de medias en procrastinación. Los resultados de dicha comparación se presentan en las Tablas 3 y 4, para niños y niñas, respectivamente. 
Tabla 3. Nivel de procrastinación percibida por los padres según los años de experiencia en actividades extraescolares de distintos tipos. Pruebas $t$ de Student.

Submuestra de alumnos (de género masculino; $\mathbf{n}=206$ ).

\begin{tabular}{llcccc}
\hline $\begin{array}{l}\text { Actividades } \\
\text { extraescolares }\end{array}$ & $\begin{array}{l}\text { Medias y desviaciones están- } \\
\text { dar por grupo }\end{array}$ & $n$ & $t$ & $d$ & $p$ \\
\hline Arte & $\begin{array}{l}\text { 0 años }=6.25(1.74) \\
+ \text { de } 0 \text { años }=6.28(1.18)\end{array}$ & $\begin{array}{c}163 \\
43\end{array}$ & .12 & -.03 & .91 \\
\hline Deporte & $\begin{array}{l}0-3 \text { años }=6.52(1.77) \\
+ \text { de } 3 \text { años }=6.01(1.48)\end{array}$ & $\begin{array}{c}98 \\
108\end{array}$ & 2.24 & .51 & $.03^{*}$ \\
\hline \multirow{2}{*}{ Otras } & $0-3$ años $=6.33(1.67)$ & 166 & 1.30 & .38 & .20 \\
& + de 3 años $=5.95(1.45)$ & 40 & & & \\
\hline \multirow{2}{*}{ Totales } & $0-6$ años $=6.37(1.76)$ & 115 & 1.23 & .28 & .22 \\
\hline
\end{tabular}

$* p<.05$

En la Tabla 3 se puede observar que, en la submuestra de alumnos de género masculino, al aumentar los años de experiencia en extraescolares de deporte disminuye el nivel de procrastinación reportado por los padres. Así pues, los resultados demuestran que las extraescolares de deporte resultan beneficiosas de cara al control de la procrastinación cuando el alumno tiene una experiencia al menos de 4 años en esta actividad. Por otro lado, en el caso de los niños (repetimos, de género masculino), tener más años de experiencia en extraescolares de arte, otras (pero no de arte o deporte) y totales no contribuye a mejorar -o empeorar, como postularía la Over-Scheduling Hypothesis- su procrastinación.

Para comprobar si es la edad la causante de estas diferencias en la procrastinación percibida por los padres calculamos una $t$ de Student comparando la media en esta característica de los 100 niños (de género masculino) de ciclo medio, que es igual a 6.24 (desviación = 1.63), con la de los 106 niños de ciclo superior, que da un resultado de 6.26 (desviación = 1.65). Esta $t=.11, p=.92, d=.02$, garantiza que la edad no tiene efecto sobre la procrastinación percibida por los padres de los alumnos. Asimismo se ha calculado la misma prueba, con idéntico resultado, para los alumnos de escuelas públicas y concertadas. La $t$ de Student es de $.61, p=.51, d=.17$, lo que indica que el tipo de escuela tampoco es significativo para el nivel de procrastinación. La media de procrastinación informada para los niños de la escuela pública es de 6.37 (desviación = 1.87) mientras que las de los niños de la escuela concertada es de 6.20 (desviación $=1.54)$. 
Por tanto, de las variables estudiadas en el presente trabajo, los 4 años o más de duración de extraescolares de deporte se mantienen como determinantes de un mejor control de la procrastinación informada por los padres de los alumnos de género masculino, en los ciclos medio y superior de educación primaria. Por otro lado, para las alumnas de género femenino el panorama es bien distinto. Los resultados y la comparación de medias que se han obtenido con este subgrupo de estudiantes se hallan en la Tabla 4.

Tabla 4. Nivel de procrastinación percibida por los padres según los años de experiencia en actividades extraescolares de distintos tipos. Pruebas t de Student. Submuestra de alumnas (de género femenino, $n=211$ ).

\begin{tabular}{llcccc}
\hline $\begin{array}{l}\text { Actividades } \\
\text { extraescolares }\end{array}$ & $\begin{array}{l}\text { Medias y desviaciones están- } \\
\text { dar por grupo }\end{array}$ & $n$ & $t$ & $d$ & $p$ \\
\hline Arte & $\begin{array}{l}\text { 0-3 años }=5.71(1.57) \\
+ \text { de } 3 \text { años }=5.09(2.02)\end{array}$ & $\begin{array}{c}168 \\
43\end{array}$ & 2.18 & .62 & $.03^{*}$ \\
\hline \multirow{2}{*}{ Deporte } & $0-3$ años $=5.56(1.69)$ & 170 & -.51 & -.15 & .61 \\
& + de 3 años $=5.71(1.76)$ & 41 & & & \\
\hline \multirow{2}{*}{ Otras } & $0-3$ años $=5.54(1.74)$ & 166 & -1.06 & -.30 & .29 \\
& + de 3 años $=5.84(1.35)$ & 44 & & & \\
\hline \multirow{2}{*}{ Totales } & $0-7$ años $=5.59(1.64)$ & 155 & -.16 & -.04 & .87 \\
\hline
\end{tabular}

$* p<.05$

Los resultados de la Tabla 4 ponen de manifiesto que, en las niñas de ciclo medio y superior de primaria, la experiencia en años de extraescolares de arte ayuda a controlar la procrastinación, según la perciben los padres. Específicamente, las niñas que cuentan con 4 años o más de asistencia a este tipo de extraescolares procrastinan significativamente menos, de acuerdo a la información de los padres, que las que no tienen esta experiencia. En cambio, la duración de la extraescolares de deporte no influye en la procrastinación percibida para las niñas ni tampoco la de las extraescolares diversas (aparte de las de arte y deporte) o totales. Así pues, el efecto beneficioso para la procrastinación percibida por los padres que en los alumnos de género masculino ejercían las actividades de deporte, en el caso de las alumnas de género femenino lo desempeñan las extraescolares artísticas.

Asimismo la edad, igual que en el grupo de niños, no influye en la procrastinación informada por los padres de las niñas, pues la $t=1.24, p=.22, d=.29$, entre las 107 niñas de ciclo medio, con una procrastinación media de 5.73 (desviación = 1.72), y las 104 niñas de 
ciclo superior, con un índice de procrastinación percibido por los padres de 5.44 (desviación = 1.64), no arroja ningún resultado significativo. Tampoco el tipo de escuela, pública o concertada, ha resultado ser de interés para nuestra variable dependiente, que arroja una $t=.64, p=$ $.52, d=.15$, con una media de procrastinación percibida por los padres de las niñas de la escuela pública de 5.68 (desviación $=1.81)$, y una media de la escuela concertada de 5.53 (desviación $=1.60$ ).

\section{Discusión y conclusiones}

La literatura sobre la influencia de la participación en actividades extraescolares en la infancia es actualmente amplia y diversa. Sin embargo, ningún trabajo ha relacionado dichas actividades con la procrastinación escolar de los alumnos de primaria. El que ahora presentamos se ha marcado este objetivo y ha encontrado diferencias significativas entre niños y niñas en edad escolar. El sentido de las diferencias indica que, de acuerdo a la información de los padres, los niños -varones- procrastinan más y hacen más extraescolares totales y de deporte que las niñas, y también que los alumnos de género masculino que han participado durante 4 años o más en extraescolares deportivas presentan un nivel significativamente más bajo de procrastinación académica percibida por los padres que sus compañeros que no tienen esta experiencia. Por otra parte, nuestros resultados asimismo muestran que, según lo que reportan los padres, las alumnas procrastinan menos y participan más en actividades extraescolares de arte que los alumnos de género masculino, y que las niñas con una duración de 4 años o más en actividades artísticas son vistas por sus progenitores como significativamente menos procrastinadoras que las que no han participado en dichas actividades. Además, los datos de nuestro estudio indican que la duración o experiencia de los alumnos y alumnas de los ciclos medio y superior de primaria en extraescolares diversas -aparte de deporte y arte- y totales no influyen ni para bien ni para mal en la procrastinación académica percibida por los padres de los estudiantes. En la misma línea, ni el hecho de cursar ciclo medio o superior de educación primaria ni la asistencia a un centro educativo público o concertado influyen significativamente en la procrastinación académica de los alumnos y alumnas, de acuerdo a la información que tenemos a partir de los padres. 
Respecto de estos datos, hay que comentar en primer lugar que son, en parte, similares a los de otros investigadores. Por un lado, confirman la mayor procrastinación en sujetos de género masculino (Steel y Ferrari, 2013), aunque de hecho, no hay datos con alumnos tan jóvenes, y, también, la alta y continuada participación de la mayoría de los alumnos en actividades extraescolares en las sociedades occidentales (Fredricks, 2012). Por el otro, reafirman la idea que defiende que la participación en extraescolares resulta beneficiosa, en general, para el bienestar psicológico de los alumnos (Keser, Akar y Yildrim, 2011; Mahoney y Vest, 2012; Ritchie y Williamon, 2011). Esto es así porque la participación prolongada en el tiempo en ciertas actividades extraescolares disminuye la procrastinación académica percibida por los padres, y se ha demostrado que a menor procrastinación, más satisfacción con uno mismo (Dewitte y Lens, 2000, entre otros). En este sentido, también hay que añadir que los resultados aquí presentados no abonan la Over-Scheduling Hypothesis sino todo lo contrario, ya que demuestran, como hemos dicho, por un lado que un cierto tiempo -4 años concretamente- de participación en determinadas actividades extraescolares -deporte para los niños y arte para las niñas- ayuda a controlar la procrastinación, y por el otro, que ni los alumnos ni las alumnas que presentan una muy larga duración en extraescolares totales presentan, de acuerdo de nuevo a la opinión de los padres, una mayor o menor procrastinación académica que sus iguales. Así pues, los resultados del presente trabajo están en la línea de los de autores que recientemente han refutado la Over-Scheduling (como Fredricks, 2012 o bien Mahoney y Vest, 2012) y rebaten a los expertos que están de acuerdo con dicha propuesta (como Shoum y Brown, 2006). O, más exactamente, nuestros resultados matizan la idea de que es la participación simultánea en muchas extraescolares la que es perjudicial, pero no la duración o continuidad en las mismas, que en ningún caso ha resultado ser dañina para el alumno, al menos en lo que a la procrastinación relatada por los padres se refiere.

Además, los hallazgos del presente trabajo confirman y amplían los de Moriana y su equipo con alumnos españoles (Moriana et al., 2006), que afirmaban que los estudiantes de primer ciclo de ESO que participaban en actividades extraescolares mixtas, o sea deportivas y cognitivas (aquí los autores incluían las clases particulares y de apoyo, que nosotros no hemos considerado), obtenían mejores notas académicas. Este resultado es en parte coincidente con el nuestro ya que diversos autores (por ejemplo, Lakshminarayan, Potdar y Reddy, 2013) han demostrado que la procrastinación académica se relaciona estrecha y negativamente con las calificaciones escolares. Así, cabe concluir que nuestro estudio está de acuerdo con el de Mo- 
riana en que, a modo general, a mayor duración de la actividad extraescolar menor procrastinación percibida por los padres -lo que implicaría mejores notas en la escuela-.

Pero por otro lado, nuestros resultados introducen, en primer lugar, la diferenciación entre géneros, en la línea de lo publicado por McCoy y su equipo (2012, que ya hemos mencionado en la Introducción), que ha resultado ser crucial para entender cómo se pueden beneficiar los niños y las niñas de cierto tipo de actividades extraescolares. Respecto de este punto, a primera vista podría pensarse que cada género saca partido del tipo de extraescolares que realiza con más frecuencia -los niños del deporte y las niñas del arte- pero esto no es exactamente así porque tal como muestra la Tabla 2, la afición extraescolar de las niñas al deporte y a las artes tiene la misma intensidad y, en cambio, las alumnas de género femenino de los ciclos medio y superior de primaria no obtienen ganancias de cara a controlar la procrastinación académica con la participación continuada en actividades deportivas, sino exclusivamente con la participación y continuidad de las extraescolares de arte y música. Este hallazgo nos parece realmente interesante y novedoso y creemos que será de utilidad para orientar a maestros y padres sobre qué tipo y duración de actividades extraescolares son aconsejables para cada alumno y alumna durante la educación primaria, principalmente en aquellos casos en que niños y niñas ya muestran una cierta tendencia hacia la demora injustificada en la realización de sus tareas de estudio.

Y, en segundo lugar pero no menos importante, el interés del estudio que ahora presentamos está en que pone de relieve que lo que resulta favorable para el control de la procrastinación no es solamente la participación en extraescolares sino la duración o experiencia continuada en las mismas. Así, tanto los niños con el deporte como las niñas con la pintura y la música, se ven beneficiados con dichas actividades a partir de una práctica de 4 años o cursos escolares, y no antes. Este hallazgo es acorde con la definición de procrastinación. Algunos autores insisten en que es un hábito que se adquiere con el tiempo (Steel, 2011), como también necesita tiempo el desarrollo de los lóbulos frontales en el cerebro, responsables del control de los impulsos más primarios que se relacionan con la capacidad de doblegar esta conducta procrastinadora o de demora irracional. De acuerdo a este argumento, parece lógico que se necesite un cierto tiempo de práctica en una actividad determinada para instaurar un hábito que ayude a controlar la procrastinación. Además, como hemos dicho, otros expertos (de Bruin et al., 2012; Dewitte y Lens, 2000; Rhodes y Dickau, 2012), conceptualizan la procrastinación como un déficit en el control del tiempo. En este caso, nuestros datos demuestran 
que a los alumnos con experiencia y continuidad en extraescolares, a pesar de que están más ocupados, no les falta tiempo para hacer las tareas escolares sino todo lo contrario, pues todo parece indicar que los niños que hacen deporte y las niñas que se dedican a la música o a la pintura, con constancia, han aprendido a distribuirlo mejor.

Asimismo, nos parece relevante el resultado que pone de relieve que, ni el ciclo educativo que cursan los alumnos y alumnas, ni el tipo de escuela al que acuden, son de importancia para la procrastinación académica percibida por los padres. Como hemos dicho, no tenemos resultados anteriores llevados a cabo con niños de esta edad y que hayan tenido en cuenta estas variables. De los estudios mencionados en la Introducción, el de Lay y colaboradores (1996) es el único que se ha realizado con alumnos tan jóvenes como los nuestros, y no trata los aspectos que aquí se han estudiado, pues centra el interés en la relación entre personalidad y procrastinación. Otros estudios, la mayoría, preguntan a estudiantes adolescentes y universitarios sobre su procrastinación académica, y relacionan esta variable, como hemos dicho, con el nivel sociocultural de las familias (Rosário et al., 2009) y con sus pautas educativas (Xin-yi Ling et al., 2011). Que sepamos pues, en ningún caso se ha analizado la influencia de la edad de los alumnos y alumnas de primaria, ni tampoco la significación del tipo de escuela, sobre la procrastinación académica. Y ambas variables son ciertamente de interés, ya que nuestro trabajo indica que la demora injustificada en los estudios aparece en edades bien tempranas, o al menos los padres lo perciben así, y no depende del ciclo de primaria que los niños y niñas estén cursando. Además, la no diferenciación entre escuela pública y concertada en cuanto a la procrastinación académica puede ayudar a aumentar la confianza de las familias hacia la calidad educativa de las instituciones públicas, a nuestro entender, a menudo injustamente desprestigiadas en nuestro país.

Para acabar, cabe añadir que en trabajos posteriores sería necesario desarrollar un método más cercano al alumno, que no precise de la apreciación de terceros, para conocer la procrastinación académica en la escuela primaria. Como es sabido, actualmente existe una gran variedad de escalas y cuestionarios, con validez sólidamente contrastada para medir la procrastinación. Aquí podemos mencionar las propuestas más clásicas, como la Procrastination Assessment Scale Students PASS (de Solomon y Rothblum, 1984), y las más actuales, como las fórmulas propuestas por Steel en su interesante página web (www.procrastinus.com) y su libro The Procrastination Equation (2011), y también la escala EDA -Escala de Demora Académica- que nosotros mismos hemos elaborado con estudiantes en Barcelona (España; 
Clariana y Martín, 2008). No obstante, todos estos instrumentos son adecuados para conocer la procrastinación académica de alumnos a partir de educación secundaria y ninguno de ellos ha sido pensado para niños más pequeños. A este respecto, quizás la observación directa de los niños, en clase y en casa estudiando y haciendo deberes, con identificadores específicos elaborados a partir de la teoría sobre el tema, sería pertinente.

A ello pensamos dedicarnos en el futuro, pues continuamos pensando que la procrastinación académica, muy a pesar de los que somos docentes y educadores, continúa siendo un tema de interés, y todavía merece la pena dedicar esfuerzos y trabajos a conocerla y a "domesticarla".

\section{Agradecimientos}

Esta investigación fue financiada con un proyecto del Ministerio de Educación, Cultura y Deporte del Gobierno de España, con ref. PK-122891, EDU2009-10651, del que fue IP la primera autora. Agradecemos sinceramente a los claustros de profesores y asociaciones de padres de las escuelas participantes su implicación en el presente trabajo. Extendemos nuestro reconocimiento también a Fernando Martín, presidente de las asociaciones de madres y padres de alumnos de las Islas Baleares (España). 


\section{Referencias}

Arisoy, P., y Tutkun, O. F.(2012). Case about orientation of primary school students to outof-school time activities. Proceedings of 3rd International Conference on New Horizons in Education (INTE), Prague, Czech Republic. Book Series, Procedia Social and Behavioral Sciences 55, 1027-1035. Amsterdam, Netherlands: Elsevier Science.

Atkinson, S., y Robson, M. (2012). Arts and health as a practice of liminality: Managing the spaces of transformation for social and emotional wellbeing with primary school children. Health \& Place, 18, 1348-1355. dx.DOI.org/10.1016/j.healthplace.2012.06.017

Bennett, P. R., Lutz, A. C., y Jayaram, L. (2012). Beyond the schoolyard: The role of parenting logics, financial resources, and social institutions in the social class cap in structured activity participation. Sociology of Education, 85(2), 131-157. DOI: $10.1177 / 0038040711431585$

Bode, H., y Hirner, V. (2013). Children with learning disabilities and handicaps in inclusive schools or in special schools? The view of parents and professionals. Klinische Padiatrie, 225(2), 57-63.

Bonhert, A., Fredricks, J. A., y Randall, E. (2010). Capturing unique dimensions of youth organized activity involvement: Theoretical and methodological considerations. Review of Educational Research, 80(4), 576-610.

Cladellas, R., Clariana, M., Badia, M., y Gotzens, C. (2013). Actividades extraescolares y rendimiento académico en alumnos de primaria. European Journal of Investigation in Health, Psychology and Education, 3(2), 87-97.

Clariana, M. (2009). Procrastinació acadèmica. Barcelona: Servei de Publicacions de la Universitat Autònoma de Barcelona, Materials 210.

Clariana, M., Gotzens, C., Badia, M., y Cladellas, R. (2012). Procrastination and cheating from secondary school to university. Electronic Journal of Research in Educational Psychology, 10(2), 737-754.

Clariana, M., Gotzens, C., y Badia, M. (2011). Continuous assessment in a large group of Psychology undergraduates. Electronic Journal of Research in Educational Psychology, 9(1), 95-112.

Clariana, M., y Martín, M. (2008). Escala de Demora Académica (EDA). Revista de Psicología General y Aplicada, 61(1), 37-51. 
de Bruin, M., Sheeran, P., Kok, G., Hiemistra, A., Prins, J., Hospers, H., y van Breukelen, G. (2012). Self-Regulatory processes mediate the intention-behavior for adherence and exercise behaviors. Health Psychology, 5. DOI: 10.1037/a0027425

Dewitte, S., y Lens, W. (2000). Procrastinators lack a broad action perspective. European Journal of Personality, 14, 121-140. DOI: 10.1002/(SICI)1099-0984(200003/04)14:2

Dimech, A. S., y Selier, R. (2011). Extra-curricular sport participation: A potential buffer against social anxiety symptoms in primary school children. Psychology of Sport and Exercise, 12, 347-354.

Elkind, D. (2001). The hurried child: Growing up too fast too soon. Cambridge, MA, US: Da Capo Press.

Ferrari, J. R., Johnson, J. L., y McCown, W. G. (1995). Procrastination and Task Avoidance: Theory, Research, and Treatment. New York: Plenum Press.

Ferrari, J. R., Özer, B., y Demir, A. (2009). Chronic procrastination among Turkish adults: Exploring decisional, avoidant, and arousal styles. The Journal of Social Psychology, 149(3), 302-308.

Fredricks, J. A. (2012). Extracurricular participation and academic outcomes: Testing the Over-Scheduling Hypothesis. Journal of Youth and Adolescence, 41(3), 295-306. DOI 10.1007/s10964-011-9704-0

Gardner, M., Roth, J., y Brooks-Gunn, J. (2011). Sports participation and juvenile delinquency: The role of the peer context among adolescent boys and girls with varied histories of problem behavior. Sport, Exercise, and Performance Psychology, 1, 19-37.

Gawrolow, C., Morgenroth, K., Schultz, R, Oettingen, G., y Gollwitzer, P.M. (2013). Mental contrasting with implementation intentions enhances self-regulation of goal pursuit in schoolchildren at risk for ADHD. Motivation and Emotion, 37(1), 134-145. DOI 10.1007/s11031-012-9288-3

Ho, Y. C., Cheung, M. C., y Chan, A. S. (2003). Music training improves verbal but not visual memory: Cross-sectional and longitudinal explorations in children. Neuropsychology, 17(3), 439-450.

Keser, F., Akar, H., y Yildirim, A. (2011). The role of extracurricular activities in active citizenship education. Journal of curriculum studies, 43(6), 809-837. DOI:10.1080/00220272.2011.591433

Lachar, D., y Gruber, C. P. (2001). Personality Inventory for Children. Second Edition (PIC-2). Los Angeles, CA, US: Western Psychological Services, wps. 
Lakshminarayan, N., Potdar, S., y Reddy, S. G. (2013). Relationship between procrastination and academic performance among a group of undergraduate dental students in India. Journal of Dental Education, 77(4), 524-528.

Lay, C. H. (1997). Explaining lower-order traits through higher-order factors: the case of trait procrastination, conscientiousness, and the specificity dilemma. European Journal of Personality, 11, 267-278. DOI: 10.1002/(SICI)1099-0984(199711)11:4

Lay, C., Kovacs, A., y Danto, D. (1998). The relation of trait procrastination to the big-five factor conscientiousness: an assessment with primary-junior school children based on self-report scales. Personality and Individual Differences, 25(2), 187-193.

Lubbers, M. J., Van Der Werf, M. P., Kuyper, H., y Hendriks, A. (2010). Does homework behavior mediate the relation between personality and academic performance? Learning and Individual Differences, 20(3), 203-208. doi:10.1016/j.lindif.2010.01.005

Luthar, S. S., Shoum, K. A., y Brown, P. J. (2006). Extracurricular involvement among affluent youth: A spacegoat “ubiquitous achievement pressures"? Developmental Psychology, 42(3), 583-597.

Mahoney, J. L., Harris, A. L., y Eccles, J. S. (2006). Organized activity participation, positive youth development, and the Over-Scheduling Hypothesis. Society for Research in Child Development: Social Policy Report, 20, 1-30.

Mahoney, J. L., y Vest, A. E. (2012). The Over-Scheduling Hypothesis revisited: Intensity of organized activity participation during adolescence and young adult outcomes. Journal of Research on Adolescence, 22(3), 409-418. DOI: 10.1111/j.1532-7795.2012.00808.x

Marsh, H. W., y Kleitman, S. (2002). Extracurricular school activities: The good, the bad, and the non-linear. Harvard Educational Review, 72, 464-514.

McCoy, S., Byrne, D., y Banks, J. (2012). Too much of a good thing? gender, 'Concerted Cultivation' and unequal achievement in primary education. Child Indicators Research, 5(1), 155-178.

McHale, J. P., Vinden, P. G.,Bush, L., Richer, D., Shaw, D., y Smith, B. (2005). Patterns of personal and social adjustment among sport-involved and noninvolved urban middleschool children. Sociology of Sport Journal, 22(2), 119-136.

Moon, S. M., y Illingworth, A. J. (2005). Exploring the dynamic nature of procrastination: A latent curve analysis of academic procrastination. Personality and Individual Differences, 38, 297-309. 
Moriana, J. A., Alós, F., Alcalá, R., Pino, M. J., Herruzo, J., y Ruiz, R. (2006). Actividades extraescolares y rendimiento académico en alumnos de Educación secundaria. Electronic Journal of Research in Educational Psychology, 4(1), 35-46.

O’Connor, S., y Jose, P. E. (2012). A propensity score matching study of participation in community activities: A path to positive outcomes for youth in New Zealand? Developmental Psychology, 48(6), 1563-1569.

Osgood, D. W., Anderson, A. L., y Shaffer, J. N. (2005). Unstructured leisure in the afterschool hours. En J. L. Mahoney, R. W. Larson, y J. S. Eccles (Eds.), Organized activities as contexts of development: Extracurricular activities, after-school and community programs (pp. 45-64). Mahwah, NJ, US: Lawrence Erlbaum.

Özer, A. (2012). Procrastination: Rethinking trait models. Egitim Ve Bilim-Education and Science, 37(166), 303-317.

Rhodes, R. E., y Dickau, L. (2012). Moderators of the intention-behaviour relationship in the physical activity domain: a systematic review. British Journal of Sports Medicine. DOI: 10.1136/bjsports-2011-090411

Rice, K. G., Richardson, C. M. E., y Clark, D. (2012). Perfectionism, procrastination, and psychological distress. Journal of Counseling Psychology, 59(2), 288-302.

Ritchie, L., y Williamon, A. (2011). Primary school children's self-efficacy for music learning. Journal of Research in Music Education, 59(2), 146-161. DOI: $10.1177 / 0022429411405214$

Rosário, P., Costa, M., Núñez, J. C., González-Pienda, J., Solano, P., y Valle, A. (2009). Academic procrastination: Associations with personal, school, and family variables. The Spanish Journal of Psychology, 12(1), 118-127.

Rothblum, E. D., Solomon, L. J., y Murakami, J. (1986). Affective, cognitive, and behavioral differences between high and low procrastinators. Journal of Counseling Psychology, 33, 387-394.

Schaefer, D. R., Simpkins, S. D., Vest, A. E., y Price, C. D. (2011). The contribution of extracurricular activities to adolescent friendships: New insights through social network analysis. Developmental Psychology, 47(4), 1141-1152.

Schouwenburg, H. C. (2004). Procrastination in academic settings: General introduction. En Schouwenburg, H. C., Lay, C. H., Pychyl, T. A., i Ferrari, J. R. (eds.) Counseling the procrastinator in academic settings. Washington DC: American Psychological Association, cap1, 3-17. 
Schouwenburg, H. C., y Groenewoud, J. T. (2001). Study motivation under social temptation: Effects of trait procrastination. Personality and Individual Differences 30, 220-240.

Schouwenburg, H. C., y Lay, C. H. (1995). Trait procrastination and the Big-Five factors of personality. Personality and Individual Differences, 18, 481-490.

Solomon, L. J., y Rothblum, E. D. (1984). Academic procrastination: Frequency and cognitive-behavioral correlates. Journal of Counselling Psychology, 31, 503-509.

Sommer, L. (2011). The theory of planned behaviour and the impact of past behaviour. International Business \& Economics Research Journal, 10(1), 91-110.

Sommer, L. (2013). Exam preparation: The influence of action control, procrastination and examination experience on students' goal intention and implementation intention. Electronic Journal of Research in Educational Psychology, 11(3), 603-630. DOI: http://dx.doi.org/10.14204/ejrep.31.13057.

Steel, P. (2007). The nature of procrastination: A meta-analytic and theoretical review of quintessential self-regulatory failure. Psychological Bulletin, 133, 65-94. DOI: 10.1037/0033-2902.133.1.65

Steel, P. (2010). Arousal, avoidant and decisional procrastinatiors: Do they exist? Personality and Individual Differences, 48, 826-934. DOI:10.1016/j.paid.2010.02.025

Steel, P. (2011). The procrastination equation. Edinburgh Gate, Harlow (UK): Pearson Educational Limit.

Steel, P., y Ferrari, J. R. (2013). Sex, education and procrastination: An epidemiological study of procrastinator's characteristics from a global sample. European Journal of Personality, 27(1), 51-58. DOI: 10.1002/per.1851

Trost, K., Biesecker, G., Stattin, H., y Kerr, M. (2007). Not wanting parents' involvement: Sign of autonomy or sign of problems? European Journal of Developmental Psychology, 4, 314-331.

Tuckman, B. (1991). The development and concurrent validity of the Procrastination Scale. Educational and Psychological Measurement, 51, 473-480.

Xin-yi Ling, M., Zhang, H., Xiong, J., y Xin-li, L. L. (2011). Related study on procrastination and parental rearing patterns in college students. Chinese Journal of Clinical Psychology, 19(5), 675-680. 


\section{Anexo}

Mi hijo o hija participa o ha participado en las siguientes actividades extraescolares:

\begin{tabular}{|c|c|c|}
\hline Tipo de actividad & $\begin{array}{l}\text { Horas a la } \\
\text { semana }\end{array}$ & $\begin{array}{l}\text { Años de práctica } \\
\text { sin contar el actual }\end{array}$ \\
\hline \multicolumn{3}{|l|}{ Deportiva } \\
\hline \multicolumn{3}{|l|}{ Hockey } \\
\hline \multicolumn{3}{|l|}{ Natación } \\
\hline \multicolumn{3}{|l|}{ Futbol } \\
\hline \multicolumn{3}{|l|}{ Atletismo } \\
\hline \multicolumn{3}{|l|}{ Gimnasia } \\
\hline \multicolumn{3}{|l|}{ Excursionismo } \\
\hline \multicolumn{3}{|l|}{ Artes marciales } \\
\hline \multicolumn{3}{|l|}{ Baloncesto } \\
\hline \multicolumn{3}{|l|}{ Tenis } \\
\hline \multicolumn{3}{|l|}{$\begin{array}{l}\text { Otras. Por favor escribir el } \\
\text { nombre de la actividad. }\end{array}$} \\
\hline \multicolumn{3}{|l|}{ Artística } \\
\hline \multicolumn{3}{|l|}{ Música } \\
\hline \multicolumn{3}{|l|}{ Ballet } \\
\hline \multicolumn{3}{|l|}{ Pintura } \\
\hline \multicolumn{3}{|l|}{ Teatro } \\
\hline \multicolumn{3}{|l|}{$\begin{array}{l}\text { Otras. Por favor escribir el } \\
\text { nombre de la actividad. }\end{array}$} \\
\hline \multicolumn{3}{|l|}{$\begin{array}{l}\text { Otras actividades extraesco- } \\
\text { lares no incluidas en las dos } \\
\text { categorías anteriores. Por } \\
\text { favor escribir el nombre de la } \\
\text { actividad. }\end{array}$} \\
\hline & & \\
\hline & & \\
\hline & & \\
\hline & & \\
\hline
\end{tabular}

Figura 1. Registro utilizado en el estudio para recoger información sobre la participación de los niños y niñas de primaria en actividades extraescolares 